\title{
Mental Disorders and Psychosocial Functioning
}

\section{Redondo de Freitas L*}

Federal University of Sao Paulo, Brazil, Sao Paulo

*Corresponding author: Luciene Redondo de Freitas, Federal University of Sao Paulo, Rua Pedro de Toledo, 669 - $3^{\circ}$ andar fundos, SP, Brazil, Tel: 5511972544255; Email: luredon@yahoo.com.br

\section{Commentary \\ Volume 2 Issue 1}

Received Date: December 11, 2017

Published Date: April 10, 2018

DOI: $10.23880 / \mathrm{mhrij}-16000116$

\section{Abstract}

The term psychosocial deficiency is characterized by the classification of a problem in the mental functions of an individual, which may be one or more limitations of the capacities of consciousness, learning, social interaction, temperament, energy and impulse, bonding, personality, memory, concentration, language, perception, according to the classification of mental functions by the CIF. The impacts of a mental disorder on a person's life are often devastating and only with the support of multiprofessional staff and good family and psychosocial support are it possible to control and overcome. However, the marks that the rupture of a state of health regarded as normal, for the transition in a diagnosed mental disorder frame bring in themselves a historical context of losses, frustrations and fragilities that are sometimes stigmatizing and excluding. Individuals with chronic and persistent mental disorders, such as schizophrenia, bipolar disorder and severe depression, present functional and intellectual impairments that compromise their ability to perform activities and performance. The promotion of the Brazilian Inclusion Law to people with severe mental disorders, recognizing them as people with psychosocial disabilities, provides an opportunity for people with mental disorders to have their fundamental rights and guarantees, equality of access and participation that favor the process of inclusion and psychosocial rehabilitation for the strengthening of autonomy, quality of life and community bonds.

\section{Commentary}

We all experience in certain life situations feelings like anxiety, sadness, nervousness, fear, loneliness, hopelessness, feeling that someone is wanting to do us some harm, constant mood swings, etc. All these mood swings and thoughts may be understandably related to circumstances of life over which we have little control or that we cannot predict their consequences accurately. The conquest of a new job can make us anxious, the loss of a loved one can overwhelm us, experiences close to assault and violence can frighten us, ending a relationship can destabilize us. Every time we are affected by circumstances of this kind, our lives become more difficult, and this is because at these times our priorities change to what requires urgency. Thus, a person who is immersed in his work can immediately stop him if he receives a call informing that his mother is hospitalized in serious condition, for example. In this example, the urgency of the mother's state of health changes the person's focus through a wave of feelings and thoughts that make it unfeasible to resume activities at that time. 


\section{Mental Health \& Human Resilience International Journal}

This can be resolved if the person discovers that he is not treating you with anything serious, or if he intensifies if he is to discover that the case is serious.

All this is very evident, but it illustrates the essential fact: every circumstance that causes a strong emotional shock generates a wave of internal reactions with feelings and thoughts that interfere with our ability to work, relate, function and choose. With this in mind, we can approach the experiences of a person with a mental disorder.

Firstly, it is important to note that the situations described above are common and do not represent mental disorders. It is expected to react with sadness, joy or concern to life events. An emotional picture is considered pathological according to some parameters: duration, impact on occupational or social functioning and degree of suffering. This evaluation is said to be subjective, since there are no laboratory tests to confirm the diagnosis. Indirect evidence that helps us characterize a change in emotional state as pathological occurs when thought or behavior prevents a person from playing the social roles he or she has been playing or would normally have the potential to play. The social role is understood as everything we do to generate and represent a full and productive life: work, study, family, friends, love relationships, life projects, life ideals, social relations, leisure, community participation, sharing of between peers. One of the main points defended by the sociologist Max Weber in 1940, when writing Theory of Organizations emphasized that "society is a set of individual actions in reciprocity: we have the experience of social actors and of the social universe itself, that is, cultural and social systems in which human beings are inserted. Thus, man establishes itself in the world from the exchange of meanings and meanings that organize the environment."

It all depends on the person's functional performance for her and for others. As stated above, life situations may well undermine this ability to perform, but in the case of mental disorders, performance capacity is not restored by the end of the situation, or the person remains with difficulty even if no situation has occurred justify it. In addition, in mental disorder, symptoms occur more frequently, intensely, and have a greater impact on one's life. Thus, a person with no disorder may well stay up all night concerned about a meeting that will occur the next day, whereas a person with panic disorder may have an acute anxiety crisis to the point of feeling short of breath and sensation of imminent death on a normal day off. In such cases, one also loses the normal capacity for selfregulation, so that unpleasant feelings and thoughts become uncontrollable, exaggerated, and inopportune. Therefore, what demarcates the limit of "normality" in mental disorders is the intensity of the suffering and symptoms, the frequency and situation in which they appear, and especially the impact it causes on the social and functional performance of the person. The Diagnostic and Statistical Manual for Mental Disorders (5th edition) defines mental disorder as follows: "A mental disorder is a syndrome characterized by a clinically significant disturbance in cognition, emotional regulation, or behavior of an individual that reflects a dysfunction in the psychological, biological or developmental processes underlying mental functioning. "(Manual DSM-5, page 62, ABP 2014 Artmed). Since 1993, mental disorders have been inserted in the 10th. Revision of the International Classification of Diseases (ICD-10), since it is understood that diseases related to mental health are also the cause of aggravation of the functions of the individual's body and that in certain cases, the consequences of death, incapacity and performance limitation.

According to the World Health Organization, published in 2010 , it is estimated that by 2020 , depression is the main disease that will incapacitate people to work (The World Health Report 2001. Mental Health: New Understanding, New Hope). Currently, mental disorders are the main causes of suicide, incapacity for work and are directly related to many situations of violation of social rights and vulnerabilities. The causes of a mental disorder can be both internal and external to the individual. For more severe conditions such as schizophrenia and bipolar disorder, there seems to be an interaction between genetic and environmental factors. It is important to highlight that the genetic component, in most cases, does not determine, but increases vulnerability. For example, having a first degree relative with schizophrenia may increase the chance to develop the disorder for up to $10 \%$, but still, the greatest chance is not to develop the disease. In other disorders, such as depression and anxious pictures, there may be a greater burden on external environments or situations. Among the environmental factors already associated with the risk of developing a mental disorder, we highlight: lifestyle, socio-cultural context, drug abuse, stress and traumas, low educational level, malnutrition.

Another important point is to emphasize that the role of diagnosis must be understood and taken care of. It aims to help understand the case and critically evaluate the best therapeutic interventions. It is often more important 


\section{Mental Health \& Human Resilience International Journal}

for professionals than for the person with the mental disorder. In this same sense, one should always emphasize the preponderance of the individual over the disease. To look at the individual more than the disorder that he has, is to give him the possibility of overcoming and opportunity to be protagonist of his own history.

Treatments vary according to the disorder and its severity. The outpatient, community-based treatment is preferred in a multidisciplinary team.

The goal of treatment is to promote overcoming the disease. The use of medications is necessary in some disorders. In Schizophrenia, for example, most patients will require pharmacological treatment. Meanwhile, pictures such as autism and mild forms of depression and anxiety disorders, have primary indication of psychosocial interventions. The combination of pharmacological and psychosocial strategies usually increases the chances of overcoming. Treatment can be understood as a process of creating opportunities for overcoming. This process is not exclusive to the mental health team and is not exhausted in treatment settings, but it expands and grows in social interaction.

Even with the best available treatments, a considerable portion of people with mental disorders will persist with cognitive impairments or difficulties in engaging in work activities. It is in this context of increasing opportunities for overcoming that the definition of psychosocial disability applied to every person with a mental disorder becomes important.

Since the first UN International Convention on the Rights of Persons with Disabilities, held in 1971; countries around the world have increasingly taken steps to include and ensure equal rights and access to services for people with disabilities.

The evolution of this debate has even contributed to the fact that the International Convention on the Rights of Persons with Disabilities, promoted by the UN in 2008, is not an attribute of the person, but rather the result of her interaction with the environment.

When we speak of a chronic and persistent mental disorder, we know that the individual has come across one or more barriers that limit their equality of full participation in society, and therefore, it is considered this individual with psychosocial deficiency. Since then, the definition of the concept of the disabled has made it possible to support people suffering from chronic mental disorders, such as schizophrenia, bipolar disorder, autism, epilepsy and depression, who had hitherto been excluded from society due mainly to the social barriers imposed: stigmas, interpersonal relations, prejudice, access to health, protective factors.

Since 1980, the first version of the International Classification of Functioning published by the World Health Organization has sought to unify the understanding of the "consequence of disease" as a way of assessing the impact of the disease on a person's health condition. This new family of health classifications, complementary to the ICD, has contributed to the collection and registration of statistical data, to measure results and quality of life, to plan social and educational policies, and to make treatment compatible with the specific conditions of the individual, considering their professional skills, rehabilitation and results (CIF, 2004, p.9, WHO).

The CIF defines deficiencies as problems in functions (mental, sensory, voice, cardiovascular, hematological, respiratory, urinary, digestive, reproductive, etc.) or body structures such as major deviation or loss.

Thus, the term psychosocial deficiency is characterized by the classification of a problem in the mental functions of an individual, which may be one or more limitations of the capacities of consciousness, learning, social interaction, temperament, energy and impulse, attention, memory, concentration, language, perception, according to the classification of mental functions by the ICF (Chapter 1, 2004).

Its evaluation is made from the identification of the fragilities and limitations that the environmental ones provide, that is, the social and attitudinal barriers; and aspects of the structure and functions of the body and mind that, due to the existence of a mental disorder, directly affect the capacity of performance and functionality of the individual within the parameters of normality to the exercise of community life.

Mental disorders occupy five positions in the ranking of the ten main causes of disability (WHO-Brasília, 2003. Innovative care for chronic conditions: structural components of action). The difficulties faced by the patient and his / her family members permeate the spheres of social and occupational life. (Between reason and illusion, São Paulo, 2008, Assis JC). For many, resuming everyday life before illness, is almost unlikely. This is because for many, lack of credibility on the part of 


\section{Mental Health \& Human Resilience International Journal}

their bodies, or by self-stigma, or even, by discrimination and ignorance of the community in which he is inserted, about the disorder he has. As a consequence, social and attitudinal barriers aggravate human functionality, impacting on performance for activities, community participation.

The impacts of a mental disorder on a person's life are often devastating and only with the support of multiprofessional staff and good family and psychosocial support is it possible to control and overcome. However, the marks that the rupture of a state of health regarded as normal, for the transition in a diagnosed mental disorder frame bring in themselves a historical context of losses, frustrations and fragilities that are sometimes stigmatizing and excluding. 